\title{
The peptide transport system Opt is involved in both nutrition and environmental sensing during growth of Lactococcus lactis in milk
}

\author{
Correspondence \\ Danièle Atlan \\ daniele.atlan@univ-lyon1.fr
}

Received 11 January 2011

Revised 3 March 2011

Accepted 3 March 2011

\author{
Mauld Lamarque, ${ }^{1}$ Dominique Aubel, ${ }^{1}$ Jean-Christophe Piard, ${ }^{2}$ \\ Christophe Gilbert, ${ }^{1}$ Vincent Juillard ${ }^{2}$ and Danièle Atlan ${ }^{1}$
${ }^{1}$ Université de Lyon, CNRS UMR 5240, Université Lyon 1, 10 rue Dubois F-69622 Villeurbanne, France Cedex, France \\ 2UMR1319 MICALIS, INRA, Centre de Recherches de Jouy-en-Josas, 78352 Jouy-en-Josas
}

\begin{abstract}
Lactococcus lactis is known to take up extracellular peptides via at least three distinct peptide transporters. The well-described oligopeptide transporter Opp alone is able to ensure the growth of $L$. lactis in milk, while the di- and tripeptide transporter DtpT is involved in a peptide-dependent signalling mechanism. The oligopeptide Opt transporter displays two peptide-binding proteins, OptA and OptS. We previously demonstrated that OptA-dependent transport is dedicated to nutritional peptides, as an optABCDF mutant (of a strain devoid of Opp) has an impaired capacity to grow in milk. Using isogenic peptide transport mutants, this study shows that biosynthesis of the Opt transporter is much less sensitive to downregulation that is dependent on extracellular peptides taken up by DtpT than is Opp biosynthesis; this peptide-dependent regulation relies on the transcriptional repressor CodY. We demonstrate the dual function of the Opt system; while OptA contributes to the bacterial nutrition during growth in milk, OptS is involved in the transport of signalling peptides derived from milk and controlling opp expression. So, these results shed new light on the peptide-dependent regulation relying on two peptide transporters with different specificities: DtpT and Opt (via OptS).
\end{abstract}

\section{INTRODUCTION}

Peptide transport plays a major role in bacteria, and more particularly in nutrition and signalling. Extracellular signalling peptides allow bacteria to sense modifications to their environment, such as population density, and adapt to their ecological niche. Some peptides directly interact extracellularly with a two-component membrane receptor, while others require internalization via peptide transporters to control biological functions such as competence, sporulation and conjugation (reviewed by Monnet, 2003; Lazazzera, 2001). Lactococcus lactis is a nonpathogenic bacterium used in the fermentation of many dairy products. L. lactis growth in milk relies on a complex proteolytic system that provides the bacterium with essential amino acids derived from milk caseins. Casein is first hydrolysed by the cell-surface proteinase PrtP into peptides of various lengths. Some of them are taken up by oligopeptide transporters, before being further hydrolysed by a large set of intracellular peptidases (Kunji et al., 1995). Three distinct transport systems have been described in $L$. lactis: the di- and tripeptide transporter DtpT and two

Abbreviations: $\beta$ Glu, $\beta$-glucuronidase; $\mathrm{BCAA}$, branched-chain amino acids; PNPU, $p$-nitrophenyl $\beta$-D-glucuronide. oligopeptide transporters Opp and Opt. DtpT belongs to the PTR family (proton motive-force driven system); it is a 12-transmembrane domain protein encoded by a single gene. Opp is an $\mathrm{ABC}$ transporter encoded by the oppDFBCA operon, and is composed of a membrane lipoprotein OppA that binds extracellular peptides, two transmembrane proteins $\mathrm{OppB}$ and OppC, and two cytoplasmic ATPases OppD and OppF (Tynkkynen et al., 1993). Opt also belongs to the ABC transporter family but differs from Opp by the presence of two peptide-binding proteins OptS and OptA (Lamarque et al., 2004). While the optS gene is transcribed independently (M. Lamarque and D. Atlan, unpublished results), optA is part of the optABCDF operon. In L. lactis, expression of many genes of the proteolytic system was shown to be peptidedependent (Guédon et al., 2001). Transport of extracellular peptides containing branched-chain amino acids (BCAAs; Ile, Leu, Val) results in a rapid increase in the intracellular BCAA pool. BCAAs are direct co-factors of CodY, a global regulator of Gram-positive bacteria, and stimulate its binding to specific DNA sequences (CodY boxes) located in gene promoter regions (Guédon et al., 2001; den Hengst et al., 2005; Sonenshein, 2005). Consequently, expression of genes located immediately downstream of the CodY 
boxes is repressed in the presence of BCAAs. The specificity of the DtpT transporter is limited to di- and tripeptides and has so far been considered as the major pathway for the entry of peptides involved in gene regulation in L. lactis (Kunji et al., 1993; Doeven et al., 2005). For instance, Leu-Pro and Pro-Leu dipeptides, internalized via DtpT, were shown to downregulate the expression of the gene encoding the PrtP proteinase of L. lactis SK11 (Marugg et al., 1995). In contrast with di- and tripeptides, longer oligopeptides were considered to be dedicated to bacterial nutrition. Transport of nutritional oligopeptides was first described to be solely carried out by the Opp transporter (Tynkkynen et al., 1993; Kunji et al., 1995). However, the Opt oligopeptide transporter is also present in most lactococcal strains and is able to replace Opp for bacterial growth in milk (Lamarque et al., 2004).

The objectives of this study were to unravel the respective roles of the Opt binding proteins (OptS and OptA) and to determine the importance of the Opt system during $L$. lactis growth in milk compared with the oligopeptide transport system Opp involved in the nutrition and the DtpT peptide-signalling pathway.

\section{METHODS}

Bacterial strains and culture conditions. Escherichia coli $\mathrm{K}-12$ strains were grown with aeration at $37^{\circ} \mathrm{C}$ in Luria-Bertani (LB) broth (Miller, 1992). L. lactis strains constructed in the present study all derived from L. lactis IL1403, a plasmid-free strain with a $\mathrm{Prt}^{-} \mathrm{Lac}^{-}$ $\mathrm{Opp}^{-}$phenotype (Bolotin et al., 2001). L. lactis cultures were incubated at $30{ }^{\circ} \mathrm{C}$ without shaking in M17 broth (Terzaghi \& Sandine, 1975) or in chemically defined medium (CDM; Poolman \& Konings, 1988) supplemented with $0.5 \%(\mathrm{w} / \mathrm{v})$ glucose or in reconstituted $10 \%(\mathrm{w} / \mathrm{v}) \mathrm{G}$ milk (Standa Industrie). When necessary, erythromycin $\left(5 \mu \mathrm{g} \mathrm{ml}^{-1}\right.$ for L. lactis and $25 \mu \mathrm{g} \mathrm{ml}^{-1}$ for E. coli), chloramphenicol $\left(5 \mu \mathrm{g} \mathrm{ml}^{-1}\right.$ for L. lactis and $20 \mu \mathrm{g} \mathrm{ml}^{-1}$ for E. coli) or ampicillin $\left(100 \mu \mathrm{g} \mathrm{ml}^{-1}\right)$ was added to the medium.

Peptide transport. Peptide transport assays were performed at $22{ }^{\circ} \mathrm{C}$ as described previously (Charbonnel et al., 2003). Briefly, cells were grown in $\mathrm{CDM}\left(\mathrm{OD}_{650} 0.8\right)$, collected by centrifugation and washed twice in $50 \mathrm{mM} \mathrm{KH}{ }_{2} \mathrm{PO}_{4}-\mathrm{K}_{2} \mathrm{HPO}_{4} \mathrm{pH} 7.5$, and the intracellular pool of amino acids was exhausted by incubating cells in the presence of $1 \mathrm{mM} 2$-deoxy-glucose. Prior to transport experiments, cells were energized with $25 \mathrm{mM}$ glucose. Energized cells $\left(\mathrm{OD}_{650} 1.0\right)$ were incubated at $22{ }^{\circ} \mathrm{C}$ in the presence of $50 \mu \mathrm{M}$ peptide, unless otherwise stated. Peptide transport was determined by measuring the internal accumulation of the amino acids constituting the peptide under study. Amino acids were detected by RP-HPLC after pre-column derivatization with $o$-phthalaldehyde.

General DNA techniques. Lactococcal genomic DNA was extracted according to a rapid method described previously (Charbonnel et al., 2003). Plasmid DNA was purified from $2 \mathrm{ml}$ overnight cultures according to the method described by Birnboim \& Doly (1979) with an additional cell treatment with lysozyme $\left(10 \mathrm{~g} \mathrm{l}^{-1}\right)$ for $30 \mathrm{~min}$ at $37{ }^{\circ} \mathrm{C}$ before lysis. Digested plasmid DNA and PCR fragments were purified with the Geneclean turbo kit (Q-BIOgene) and QIAquick PCR purification kit (Qiagen), respectively.

Transformation of $E$. coli $\mathrm{K}-12$ and $L$. lactis IL1403 and derivatives. Electrocompetent $E$. coli cells were prepared according to the method described by Dower et al. (1988), except that the electric shock was performed at 2000 V. L. lactis IL1403 cells were treated according to the method described by Wells et al. (1993) and used immediately for electroporation, as described previously (Lamarque et al., 2004).

Construction of IL1403-derived mutants. Partial deletion $(\sim 700 \mathrm{bp})$ of each gene of interest was obtained by double-crossover homologous integration using the thermosensitive plasmid pGh5: ISS1 (Biswas et al., 1993). 5' and $3^{\prime}$ homologous regions were amplified by PCR using DyNAzymeTMEXT polymerase (Finnzymes), L. lactis IL1403 DNA as template and the specific primers listed in Table 1. The resulting PCR fragments were subcloned into PCR-Script Cam SK ${ }^{+}$ vector (Stratagene) by using HindIII-EcoRI and EcoRI-PstI restriction sites, respectively. The partially deleted genes were further subcloned into HindIII- and PstI-digested pGh9: ISS1 (which also deletes ISS1 located between HindIII and PstI sites). Following L. lactis IL1403 electroporation with modified pGh9: ISS1, clones carrying a deleted version of the gene of interest were selected according to the procedure described by Biswas et al. (1993). Mutants of optS, optA, dtpT and $\operatorname{cod} Y$ were verified by PCR amplification and sequencing.

Construction of transcriptional fusions and genomic-sitespecific integration. DNA regions of $200 \mathrm{bp}$ upstream of the SK11 oppDFBCA operon and $231 \mathrm{bp}$ upstream of the IL1403 optABCDF operon, respectively, were amplified by PCR using the primers listed in Table 1. The PCR fragments were independently cloned between the XbaI and PstI sites of plasmid pLB85 upstream of the promoterless uidA gene, encoding the E. coli $\beta$-glucuronidase ( $\beta$ Glu) as a reporter (Brøndsted \& Hammer, 1999). The recombinant vectors were subsequently checked by appropriate restriction analysis and sequencing prior to electroporation into L. lactis strains. The generation of a new attL site and the removal of the former attB site following chromosomal integration were further confirmed by PCR using the primers listed in Table 1.

Enzyme and protein assays from bacterial extracts. L. lactis cells grown in $100 \mathrm{ml} C D M$ medium (or milk) up to $\mathrm{OD}_{600} \sim 0.7-0.8$ were harvested by centrifugation, washed with $40 \mathrm{ml}$ GUS buffer $(50 \mathrm{mM}$ $\mathrm{NaHPO}_{4}, \mathrm{pH}$ 7.0) and resuspended in $4 \mathrm{ml}$ of the same buffer. After cell breakage by a French press at 20000 p.s.i. and centrifugation, the supernatants were used as soluble cellular extracts for $\beta$ Glu assays. Enzymic reactions were prepared as follows: $100 \mu \mathrm{l}$ extract, $790 \mu \mathrm{l}$ GUS buffer, $100 \mu 10 \mathrm{mM} p$-nitrophenyl $\beta$-D-glucuronide (PNPU) and $10 \mu \mathrm{l} 1 \mathrm{M} \beta$-mercaptoethanol. Time-course release of $p$ nitrophenol from PNPU was followed at $415 \mathrm{~nm}$. PepN activity was assayed with $0.3 \mathrm{mM}$ lysyl- $p$-nitroanilide as described previously (Atlan et al., 1989). The protein content was determined as described by Bradford (1976) using the Bio-Rad protein assay (Bio-Rad) with BSA as the standard.

Immunoblotting of OptA and OptS. Immunodetection of OptA and OptS was carried out as described previously (Lamarque et al., 2004). The antibody dilutions used were as follows: rabbit anti-OptA, $1: 8000$; rabbit anti-OptS, $1: 2000$; anti-rabbit immunoglobulin Gperoxidase conjugate, 1:4000 (Sigma-Aldrich). Detection was performed using Lumi-LightPlus Western blotting substrate (Roche Diagnostics).

Calculations and statistical analyses. All data are expressed as mean \pm SEM of values for three reactions, unless otherwise stated. The effect of treatments (gene inactivation, addition of peptides) on $\beta$ Glu activity was assessed using ANOVA for repeated measurements. When ANOVA indicated significant differences, treatments were compared using the Student-Newman-Keuls multiple comparison test. Statistical significance was set at $P<0.05$. Calculations were performed using the SigmaStat software package (Systat Software). 
Table 1. Primers used in this study

Restriction sites are underlined.

\begin{tabular}{|c|c|c|}
\hline Gene & Primer & Primer sequence $\left(5^{\prime}-3^{\prime}\right)$ \\
\hline \multicolumn{3}{|c|}{ Construction of deletion mutants } \\
\hline \multirow[t]{4}{*}{$\Delta o p t A$} & optAmut3 & AACTGCAGAGCATGCGGCAATAGCG \\
\hline & optArev3 & GCTCTAGAGCCAATAATCGTCATTCTTCACG \\
\hline & optAmut4 & GGAATTCATTGAGCGAAATTGGCGA \\
\hline & optA mut6 & CCCAAGCTTTTATTATTTGAGGTAAGCTGTTTTGAAGTCAT \\
\hline \multirow[t]{4}{*}{$\Delta o p t S$} & optS3Hind & CCCAAGCTTAAAACAAGTGAGCAAAAAAACATC \\
\hline & optSrdelta & CGGAATTCCCCCATCAAATAGGCATAT \\
\hline & optsensdelta & CGGAATTCACTGTCAATTTAAAACTTGTTCC \\
\hline & optSrev1 & AACTGCAGTTATTTAACATAAGCCGATTTTAGG \\
\hline \multirow[t]{4}{*}{$\Delta d t p T$} & dtpTsens1 & AACTGCAGTCGGTCAACCTCGCGGC \\
\hline & dtpTmut1 & GGAATTCTGGAGCAATCAGAGAACCCATGTT \\
\hline & dtpTmut2 & GGAATTCCCGCTCTTCATCGTTCTCTTGTCAC \\
\hline & dtpTrevl & CCCAAGCTTGACAATAAGGAGGATAATTCCCACG \\
\hline \multirow[t]{4}{*}{$\Delta \operatorname{cod} Y$} & codYsens 1 & AACTGCAGGCAAGATGGAGTGACCG \\
\hline & codYrev1 & GGAATTCACGTACTGCCGCACGAAC \\
\hline & codYsens2 & GGAATTCGACACCATGGCAACTATGGC \\
\hline & codYrev2 & CCCAAGCTTCCCTGGCTTTTAGAAATTACG \\
\hline \multicolumn{3}{|c|}{ Promoter regions } \\
\hline \multirow[t]{2}{*}{ optA } & PoptA1 & GCTCTAGAAAAACACGAACCAGTCAGAGCC \\
\hline & PoptA3 & AAAACTGCAGTTCATGAATTGTGTCTGACTTCGC \\
\hline \multirow[t]{2}{*}{$o p p D$} & PoppDSKsens & GCTCTAGAGCAATTCGGGTATAATAAAAGC \\
\hline & PoppDSKrev & AACTGCAGTGAAATCTTTCTAAATAGACTTAATAA \\
\hline \multicolumn{3}{|c|}{ Detection of $a t t B$ and $a t t L$ sites* } \\
\hline & pattBL & CTACTGCTTCACCAG \\
\hline & gusArev & GTCGAGTTTTTTGATTTCACGGG \\
\hline & BI-POBlinv & GTATGCAGCGATGTCGTTACCC \\
\hline
\end{tabular}

${ }^{\star}$ The presence of the chromosomal attB site was detected by PCR with the primer pair pattBL/BI-POBinv. Insertion of a fusion at attB generated a new site (attL) detected by PCR with the primers pattBL/gusArev.

\section{RESULTS}

\section{Extracellular peptides affect opt expression}

In L. lactis, dipeptides have been shown to affect the transcriptional pattern of genes encoding proteins of the proteolytic system. These peptides are hydrophobic, contain at least one branched-chain amino acid and are internalized into the bacteria via the di- and tripeptide transporter DtpT (Guédon et al., 2001, 2005). To investigate whether Opt expression was sensitive to peptidedependent regulation, we chose a transcriptional fusion strategy in isogenic peptide-transport mutants. We took advantage of the IL1403 strain background that did not express $O P P$ genes to focus our study on the effect of peptides solely taken up by Opt and DtpT.

The promoter regions of optS (PoptS) and the optABCDF operon (PoptA) from L. lactis IL1403, or oppDFBCA (PoppD) from L. lactis SK11 (PoppD from L. lactis IL1403 is not functional) (Lamarque et al., 2004) were cloned in the pLB85 vector, upstream of the promoterless uidA gene encoding the $E$. coli $\beta$ Glu enzyme as a reporter. Then, the resulting constructs were integrated at the attB chromosomal site of L. lactis IL1403 or in derived strains deleted for either optA, optS, $d t p T$ or $\operatorname{cod} Y$ genes (Brøndsted \& Hammer, 1999). In order to assess the effect of peptides originating from a pancreatic digest of casein (tryptone), bacteria were cultured in CDM or CDM supplemented with $1.5 \%$ tryptone $\left(\mathrm{CDM}_{\text {tryp }}\right)$ (Fig. 1). $\beta$ Glu activities were measured using PNPU as the substrate. In the parental strain IL1403, no $\beta$ Glu activity driven by PoppD could be detected in the presence of a pancreatic digest of caseins in the culture medium, which reflects a full repression of the PoppD-uidA fusion (Fig. 1a). Repression was abolished in a $\operatorname{cod} Y$ and single or multiple $d t p T$ mutants. Deletions of optA or optS did not affect $\beta$ Glu activities, suggesting that the Opt transporter was not involved in the transport of peptides affecting the transcriptional regulation of $\mathrm{P} o p p D-u i d A$. These results confirm that inhibitory peptides are taken up by DtpT and that this signalling pathway resulted in the activation of the transcriptional repressor CodY (Fig. 1a).

Analysis of PoptA-uidA expression revealed that the BGlu activity was decreased 3.6-fold in bacteria grown in $\mathrm{CDM}_{\text {tryp }}$ compared with CDM, and that this inhibition was less severe 

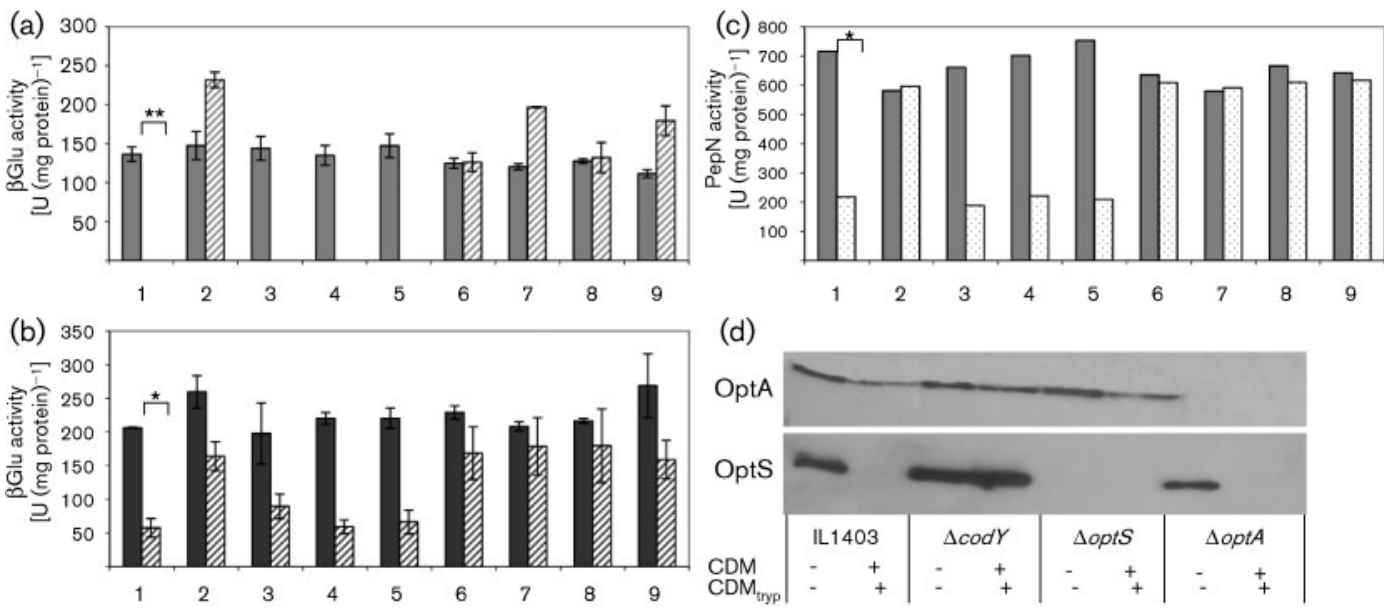

Fig. 1. Peptide- and CodY-dependent transcriptional repression of the opt system. IL1403 or derived strains were grown in CDM (solid bars) or $\mathrm{CDM}_{\text {tryp }}$ (CDM supplemented with $1.5 \%$ tryptone; hatched or dotted bars) and assayed for $\beta$ Glu activities driven by (a) PoppD or (b) PoptA. Data are the means \pm SEM of three independent experiments. Statistically significant differences ( $t$-test) are highlighted by an asterisk: ${ }^{\star} P \leqslant 0.05,{ }^{\star \star} P \leqslant 0.01$. (c) Peptidase PepN activity was used as a control for peptide-dependent regulation. 1, IL1403; 2, $\Delta \operatorname{cod} Y ; 3, \Delta o p t A ; 4, \Delta o p t S ; 5, \Delta o p t S-o p t A ; 6, \Delta d t p T ; 7, \Delta d t p T-o p t A ; 8, \Delta d t p T-$ optS; 9, $\Delta d t p T$-optS-optA. (d) Immunodetection of peptide-binding proteins OptA and OptS in IL1403 or derived strains using rabbit polyclonal antibodies. Equivalent amounts of $L$. lactis cells were loaded in each lane.

in $\operatorname{cod} Y$ or $d t p T$ mutants (Fig. 1b). This significant repression effect $(P<0.05)$ closely resembled that observed for PepN peptidase activity used as a control of the peptide-dependent regulation of the proteolytic system (Fig. 1c). To further verify that the transcriptional repression observed with the promoter-uidA fusion constructs reflected genuine protein level changes, immunodetection of peptide-binding proteins OptA and OptS was carried out after growth of IL1403 in CDM or $\mathrm{CDM}_{\text {tryp. }}$. The OptA protein level perfectly matched PoptA transcriptional regulation, i.e. there was a significant decrease of OptA biosynthesis in the presence of peptides and suppression of this effect in a $\operatorname{cod} Y$ mutant but not in an optS mutant (Fig. 1d). Interestingly, immunodetection of OptS revealed that the biosynthesis of this protein was much more sensitive to the added peptides than OptA biosynthesis. The fact that OptS downregulation in $\mathrm{CDM}_{\text {tryp }}$ cultures was abolished in a codY mutant suggests CodY-dependent regulation. Nevertheless, this hypothesis could not be confirmed at the transcriptional level by PoptS-uidA fusion, as no enzyme activity driven by PoptS could be detected under our conditions.

Thus, these results showed that biosynthesis of both $L$. lactis oligopeptide transporters (Opp and Opt) was downregulated by peptides originating from a pancreatic digest of casein and taken up by DtpT.

\section{Opt and DtpT are entry pathways for signalling peptides from milk}

Previous work on peptide-dependent regulation of proteolytic genes has been carried out using synthetic or commercial peptide mixtures (Guédon et al., 2001; Marugg et al., 1995). Nevertheless, the effect of either endogenous milk peptides or peptides resulting from casein proteolysis by the lactococcal cell-surface proteinase PrtP has not yet been addressed. As the strain IL1403 used in this study is devoid of the plasmid prtP and lac systems essential for optimal growth in milk (de Vos et al., 1989), we restored the capacity of bacterial growth in milk supplemented with glucose by transferring pNZ521, which encodes the $\mathrm{P}_{\mathrm{III}^{-}}$ type proteinase from L. lactis SK11; the resulting recombinants are hereafter indicated with "-P". As expected, the IL1403-P strain exhibited a biphasic exponential growth in milk supplemented with glucose, with the first exponential phase corresponding to the utilization of free amino acids and peptides from milk, while the second phase reflects utilization of casein-derived peptides generated by PrtP (Fig. 2a) (Juillard et al., 1995). The optA-P mutant was severely impaired for growth in milk, while deletion of optS or $d t p T$ had no significant effect. These observations confirmed previous results suggesting that OptA was involved in supplying $L$. lactis with nutritional peptides (Lamarque et al., 2004).

The plasmid pNZ521 was also transferred into isogenic peptide transport mutants harbouring transcriptional PoppD-uidA or PoptA-uidA fusions. $\beta$ Glu activities were measured in the middle of the second exponential growth phase corresponding to PrtP-dependent casein hydrolysis. Surprisingly, $\beta$ Glu activity driven by PoppD-uidA was not detected after growth of IL1403-P in milk (Fig. 2b). However, this transcriptional fusion is functional, as enzymic activity was detected after growth of the same strain in CDM. Thus, we can conclude that $o p p D$ expression was totally repressed in the IL1403-P strain grown in milk. In contrast, $\beta$ Glu activity was detected in 

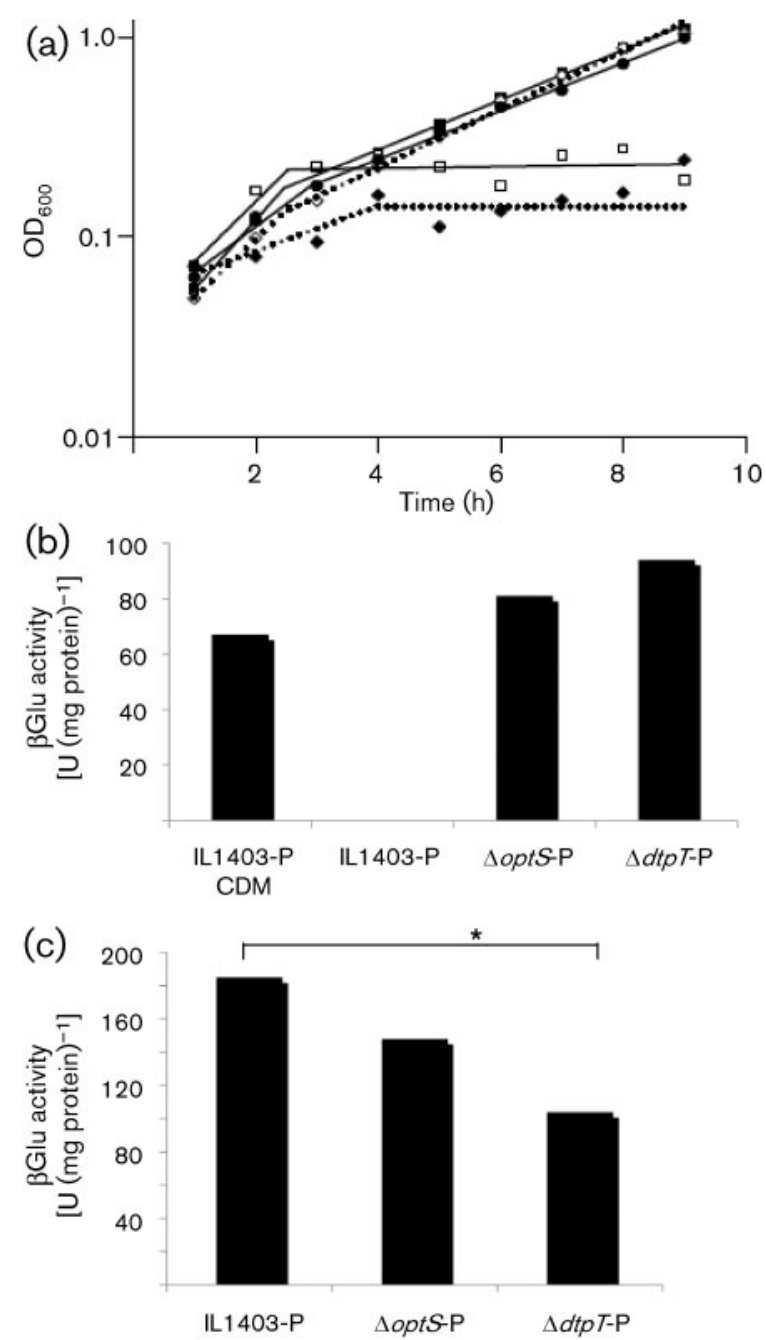

Fig. 2. DtpT and OptS are entry pathways for signalling peptides from milk. (a) Growth in milk of L. lactis IL1403 and isogenic peptide transport mutants harbouring a plasmid with the prtP gene encoding the cell-surface PrtP proteinase (-P strains): IL1403 ( $\square$ ), IL1403-P (ם), $\Delta o p t S-P(\diamond$, dotted line), $\Delta d t p T-\mathrm{P}(\bullet)$ and $\Delta o p t A-$ $\mathrm{P}(\boldsymbol{\gamma}$, dotted line). (b, c) $\beta \mathrm{Glu}$ activities of PoppD-uidA (b) or PoptA-BuidA (c) fusion after growth of IL1403 or derived strains in milk. Cells were collected at $\mathrm{OD}_{600} \sim 0.7-0.8$, corresponding to the second exponential growth phase. $\beta$ Glu activity was measured using PNPU as the substrate. Statistically significant differences ( $t-$ test) are highlighted by an asterisk: ${ }^{\star} P \leqslant 0.05,{ }^{\star \star} P \leqslant 0.01$.

strains carrying deletions of the optS or $d t p T$ genes. This result clearly shows that OptS and DtpT are responsible for the uptake of repressive peptides originating from milk casein or resulting from caseinolysis by PrtP.

Analysis of PoptA-uidA expression revealed that cultures grown in milk displayed a similar $\beta$ Glu activity to that measured in CDM (Figs $1 \mathrm{~b}$ and $2 \mathrm{c}$ ). While the optS deletion did not significantly affect the level of this activity, deletion of $d t p T$ led to a twofold decrease in $\beta$ Glu activity compared with the parent IL1403-P $(P<0.05)$. This result suggests that DtpT, so far known as a transporter of repressing peptides, might also be responsible for the uptake of activating di- and tripeptides from milk (or milk hydrolysis).

Taken together, these results expand the role of DtpT as the major transporter of signalling peptides and further validate our genetic approach. Consequently, these data show for the first time, to our knowledge, that OptS is also involved in environmental sensing by taking up repressive peptides from milk.

\section{OptS and OptA display unique peptide specificities}

Our results indicate that OptS and DtpT are main entry pathways for regulatory peptides while OptA would be involved in the uptake of nutritional peptides. We had previously analysed the transport of three peptides by optS or optABCDF interrupted mutants (Lamarque et al., 2004). Here, we took advantage of the new isogenic optA and optS single and double peptide transport mutants to further define the respective OptS- and OptA-dependent transport specificities. The strains were tested for their transport capabilities towards 17 peptides containing 2-10 amino acids. The parental IL1403 strain was able to take up peptides containing up to six residues but not longer peptides (Table 2). In contrast, an optA mutant was totally impaired in its transport capacity of tri- to hexapeptides. This tripeptide uptake ability was not expected, as so far OptA was thought to be restricted to oligopeptide transport. Absence of OptS did not affect transport rates of tri- to hexapeptides or of hydrophobic FL and MM dipeptides, which correlates with the reported DtpT specificity and highest affinity towards peptides containing at least one hydrophobic residue (Fang et al., 2000). However, the optS mutant exhibited a significant fourfold decrease in the transport rate of the EK dipeptide, suggesting that OptS might display preferences for charged dipeptides.

Taken together, these data highlight specificity differences between both peptide-binding proteins OptS and OptA, and clearly demonstrate the originality of Opt transport specificity compared with those of Opp and DtpT.

\section{DISCUSSION}

Previous studies have demonstrated the nutritional function of the oligopeptide transport system Opp in L. lactis (Kunji et al., 1995; Juillard et al., 1995). Characterization of a second oligopeptide transport system Opt raised the question of the respective role of each system in L. lactis. Our data clearly show that Opt supports a dual function, in both nutrition and signalling. Indeed, we provide evidence with IL1403 isogenic peptide transport mutants that Opt is able, via OptA, to sustain $L$. lactis growth in milk. In contrast, neither OptS nor DtpT was required for optimal 
Table 2. Initial rate of peptide transport by L. lactis IL1403 and derived strains

Peptide concentration used for transport assay was $50 \mu \mathrm{M}$, except for VGDE (300 $\mu \mathrm{M})$. Values are the mean of two independent experiments.

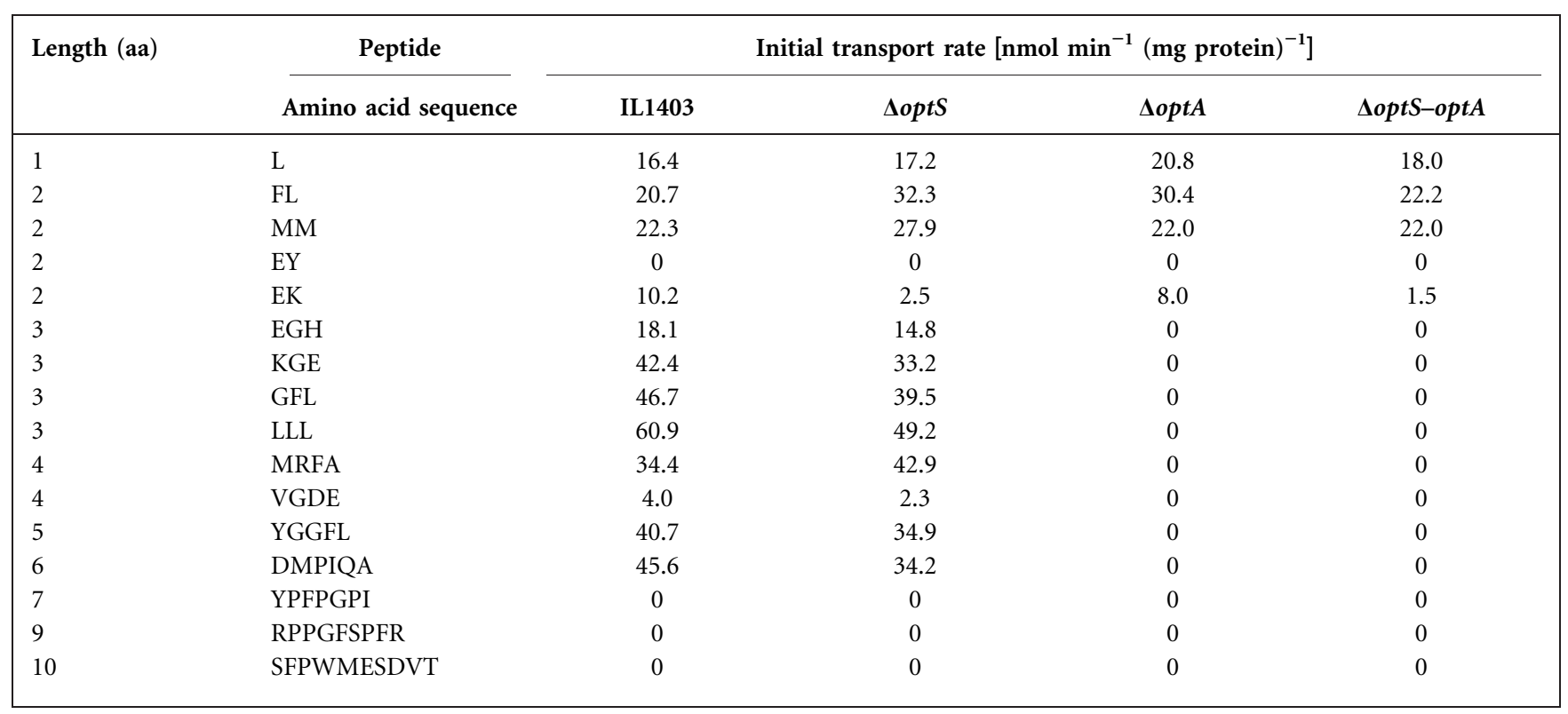

growth of IL1403-P in milk. This marked difference in nutritional function is also reflected in the difference in the substrate specificity of OptA- and OptS-dependent transport. Performing transport assays with a large panel of synthetic peptides revealed that OptA was responsible for the uptake of tri- to hexapeptides, while OptS was restricted to di-peptides at least under our experimental conditions, thereby confirming that OptA is an oligopep-

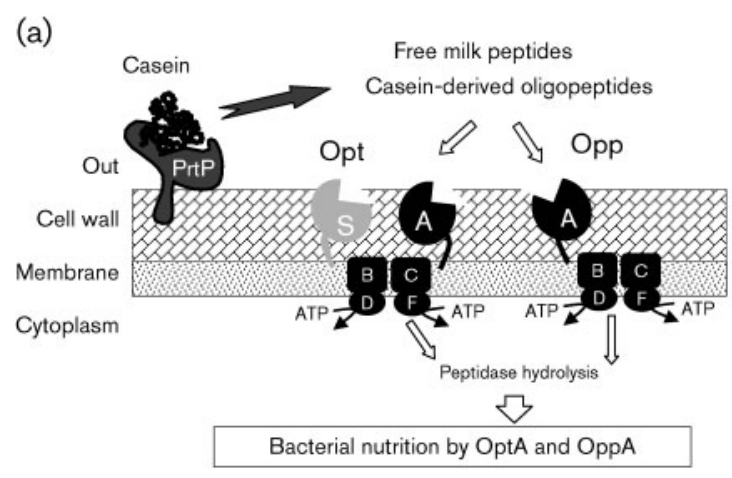

(b)

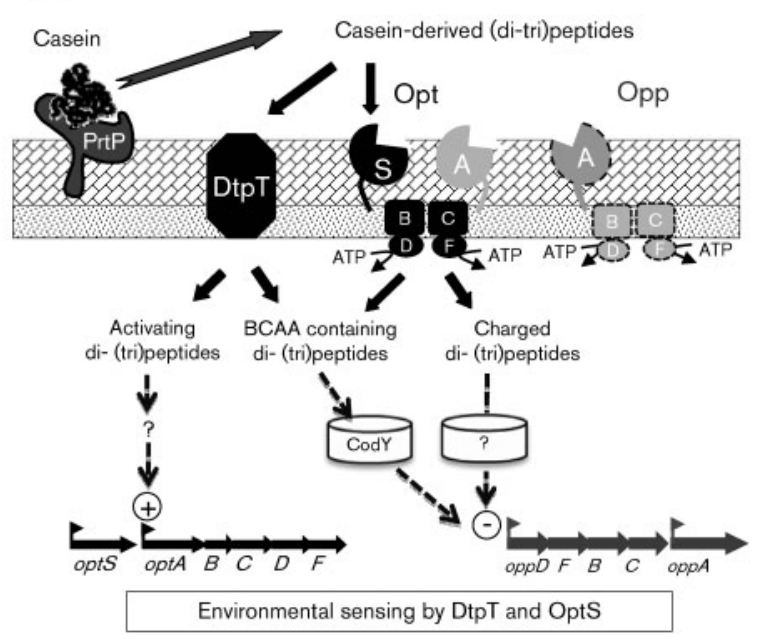

Fig. 3. Model of peptide-dependent nutrition and regulation involving the peptide transporters (Opp, Opt and DtpT) during growth of $L$. lactis in milk. (a) The nutritional function. During exponential growth in milk, the opt and opp systems are expressed. As a consequence, Opp and Opt (via OptA) transporters sustain bacterial growth with the free oligopeptides present in milk (first exponential bacterial growth phase) or those released upon casein hydrolysis by the cell envelope proteinase (PrtP) (second exponential growth phase). (b) Environmental sensing. Casein degradation results in an increased concentration of regulatory casein-derived (di-tri)peptides, which are taken up via OptS and DtpT, and leads to a progressive repression of opp expression. This mechanism is controlled by the transcriptional repressor CodY that senses the pool of BCAAs, and probably by another regulatory protein(s) not yet identified. Simultaneously, some peptides only internalized by DtpT might activate optABCDF expression by a mechanism that remains to be elucidated. 
tide-binding protein. It is worth mentioning that determination of the upper size limit for peptide transport is very challenging, as model peptides used for transport studies do not necessarily have a high enough affinity for the binding protein to be transported. In fact, we had previously shown that $L$. lactis SK11 deprived of the Opp transporter was able to transport peptides of seven or nine residues purified from milk (Lamarque et al., 2004). However, it might be possible that OptA displays a broader specificity towards natural milk peptides than to the synthetic ones used in this study. What is clear is that OptA specificity appears much more limited than that of OppA, which can bind peptides from between 4 and 35 residues (Detmers et al., 2000).

Biosynthesis of two oligopeptide transporters has been described in other bacteria. In Bacillus subtilis, for instance, Opp and App oligopeptide transporters display overlapping specificities that improve peptide transport efficiency according to environmental changes (Koide et al., 1999). The presence of multiple peptide-binding proteins encoded by genes under the control of different promoters is an advantageous strategy to broaden substrate specificity and improve adaptation capacities, as was described in Lactobacillus delbrueckii subsp. bulgaricus (Peltoniemi et al., 2002). Similarly, our results showed that during bacterial growth in CDM, OptA and OptS biosynthesis was repressed in a CodY-dependent manner by signalling peptides taken up by DtpT, but not to the same extent, since the OptS biosynthesis was much more sensitive to peptides present in the tryptone added to the culture medium. Besides, the moderate repression of the PoptAuidA fusion, compared with the full repression of PoppDuidA, could be explained by differences in the CodY box sequences located in these promoter regions (den Hengst et al., 2005). Actually, the CodY box located in the optA promoter differs from the consensus sequence by two nucleotides, whereas a unique substitution was observed in the CodY box of the oppD promoter from L. lactis SK11.

Importantly, we showed for the first time that signalling peptides, originating from milk and possibly generated by the cell-surface proteinase PrtP, are delivered into the bacteria via OptS and downregulate opp expression. Hence, the Opt transporter would support a dual function, one of nutrition via OptA and a second in environmental sensing via OptS (Fig. 3). Based on the observations that Opp biosynthesis was much more sensitive to repressive peptides than Opt and that PoppD promoter activity was not detected during the second exponential growth phase in milk, it could be supposed that Opt might be the major nutrient transporter in wild-type Lactococcus strains harbouring both transport systems, at least during certain bacterial growth phases in milk.

The peptide-dependent regulation during L. lactis growth in milk involves two peptide transporters with different specificities: DtpT and Opt (via OptS) (Fig. 3b). DtpT is able to take up di- and tripeptides from milk and those resulting from casein degradation by the Lactococcus proteinase PrtP (or a pancreatic extract such as tryptone); in contrast, di-(tri)peptides internalized by OptS are more specifically associated with Lactococcus growth in milk and seem dedicated to the downregulation of Opp. However, it is also possible that other unidentified regulation mechanisms could be set up by the bacteria to finely regulate and coordinate the expression of genes encoding different components of the proteolytic system. In support of this hypothesis, our results tend to suggest the existence of an activating mechanism of optA expression depending on diand tripeptides that would be taken up by DtpT (Fig. 3b). Future work will be necessary to fully understand the complexity of peptide transport regulation and specificity in dairy milk bacteria.

\section{ACKNOWLEDGEMENTS}

The authors thank Willem de Vos for the kind gift of pNZ521 plasmid. This work was supported by grants from Centre National de la Recherche Scientifique and the French Ministry of Education and Research.

\section{REFERENCES}

Atlan, D., Laloi, P. \& Portalier, R. (1989). Isolation and characterization of aminopeptidase-deficent Lactobacillus bulgaricus mutants. Appl Environ Microbiol 55, 1717-1723.

Birnboim, H. C. \& Doly, J. (1979). A rapid alkaline extraction procedure for screening recombinant plasmid DNA. Nucleic Acids Res 7, 1513-1523.

Biswas, I., Gruss, A., Ehrlich, S. D. \& Maguin, E. (1993). Highefficiency gene inactivation and replacement system for Grampositive bacteria. J Bacteriol 175, 3628-3635.

Bolotin, A., Wincker, P., Mauger, S., Jaillon, O., Malarme, K., Weissenbach, J., Ehrlich, S. D. \& Sorokin, A. (2001). The complete genome sequence of the lactic acid bacterium Lactococcus lactis ssp. lactis IL1403. Genome Res 11, 731-753.

Bradford, M. M. (1976). A rapid and sensitive method for the quantitation of microgram quantities of protein utilizing the principle of protein-dye binding. Anal Biochem 72, 248-254.

Brøndsted, L. \& Hammer, K. (1999). Use of the integration elements encoded by the temperate lactococcal bacteriophage TP901-1 to obtain chromosomal single-copy transcriptional fusions in Lactococcus lactis. Appl Environ Microbiol 65, 2859-2865.

Charbonnel, P., Lamarque, M., Piard, J. C., Gilbert, C., Juillard, V. \& Atlan, D. (2003). Diversity of oligopeptide transport specificity in Lactococcus lactis species. A tool to unravel the role of OppA in uptake specificity. J Biol Chem 278, 14832-14840.

den Hengst, C. D., van Hijum, S. A., Geurts, J. M., Nauta, A., Kok, J. \& Kuipers, O. P. (2005). The Lactococcus lactis CodY regulon: identification of a conserved cis-regulatory element. J Biol Chem 280, 34332-34342.

Detmers, F. J., Lanfermeijer, F. C., Abele, R., Jack, R. W., Tampé, R., Konings, W. N. \& Poolman, B. (2000). Combinatorial peptide libraries reveal the ligand-binding mechanism of the oligopeptide receptor OppA of Lactococcus lactis. Proc Natl Acad Sci U S A 97, 1248712492. 
de Vos, W. M., Vos, P., de Haard, H. \& Boerrigter, I. (1989). Cloning and expression of the Lactococcus lactis subsp. cremoris SK11 gene encoding an extracellular serine proteinase. Gene 85, 169-176.

Doeven, M. K., Kok, J. \& Poolman, B. (2005). Specificity and selectivity determinants of peptide transport in Lactococcus lactis and other microorganisms. Mol Microbiol 57, 640-649.

Dower, W. J., Miller, J. F. \& Ragsdale, C. W. (1988). High efficiency transformation of $E$. coli by high voltage electroporation. Nucleic Acids Res 16, 6127-6145.

Fang, G., Konings, W. N. \& Poolman, B. (2000). Kinetics and substrate specificity of membrane-reconstituted peptide transporter DtpT of Lactococcus lactis. J Bacteriol 182, 2530-2535.

Guédon, E., Renault, P., Ehrlich, S. D. \& Delorme, C. (2001). Transcriptional pattern of genes coding for the proteolytic system of Lactococcus lactis and evidence for coordinated regulation of key enzymes by peptide supply. J Bacteriol 183, 3614-3622.

Guédon, E., Sperandio, B., Pons, N., Ehrlich, S. D. \& Renault, P. (2005). Overall control of nitrogen metabolism in Lactococcus lactis by CodY, and possible models for CodY regulation in Firmicutes. Microbiology 151, 3895-3909.

Juillard, V., Le Bars, D., Kunji, E. R., Konings, W. N., Gripon, J. C. \& Richard, J. (1995). Oligopeptides are the main source of nitrogen for Lactococcus lactis during growth in milk. Appl Environ Microbiol 61, 3024-3030.

Koide, A., Perego, M. \& Hoch, J. A. (1999). ScoC regulates peptide transport and sporulation initiation in Bacillus subtilis. J Bacteriol 181, 4114-4117.

Kunji, E. R., Smid, E. J., Plapp, R., Poolman, B. \& Konings, W. N. (1993). Di-tripeptides and oligopeptides are taken up via distinct transport mechanisms in Lactococcus lactis. J Bacteriol 175, 2052 2059.

Kunji, E. R., Hagting, A., De Vries, C. J., Juillard, V., Haandrikman, A. J., Poolman, B. \& Konings, W. N. (1995). Transport of $\beta$-caseinderived peptides by the oligopeptide transport system is a crucial step in the proteolytic pathway of Lactococcus lactis. J Biol Chem 270, $1569-1574$.
Lamarque, M., Charbonnel, P., Aubel, D., Piard, J.-C., Atlan, D. \& Juillard, V. (2004). A multifunction ABC transporter (Opt) contributes to diversity of peptide uptake specificity within the genus Lactococcus. J Bacteriol 186, 6492-6500.

Lazazzera, B. A. (2001). The intracellular function of extracellular signaling peptides. Peptides 22, 1519-1527.

Marugg, J. D., Meijer, W., van Kranenburg, R., Laverman, P., Bruinenberg, P. G. \& de Vos, W. M. (1995). Medium-dependent regulation of proteinase gene expression in Lactococcus lactis: control of transcription initiation by specific dipeptides. J Bacteriol 177, 2982-2989.

Miller, J. (1992). A Short Course in Bacterial Genetics: a Laboratory Manual and Handbook for Escherichia coli and Related Bacteria. Cold Spring Harbor, NY: Cold Spring Harbor Laboratory Press.

Monnet, V. (2003). Bacterial oligopeptide-binding proteins. Cell Mol Life Sci 60, 2100-2114.

Peltoniemi, K., Vesanto, E. \& Palva, A. (2002). Genetic characterization of an oligopeptide transport system from Lactobacillus delbrueckii subsp. bulgaricus. Arch Microbiol 177, 457-467.

Poolman, B. \& Konings, W. N. (1988). Relation of growth of Streptococcus lactis and Streptococcus cremoris to amino acid transport. J Bacteriol 170, 700-707.

Sonenshein, A. L. (2005). CodY, a global regulator of stationary phase and virulence in Gram-positive bacteria. Curr Opin Microbiol 8, 203-207.

Terzaghi, B. E. \& Sandine, W. E. (1975). Improved medium for lactic streptococci and their bacteriophages. Appl Microbiol 29, 807-813.

Tynkkynen, S., Buist, G., Kunji, E., Kok, J., Poolman, B., Venema, G. \& Haandrikman, A. (1993). Genetic and biochemical characterization of the oligopeptide transport system of Lactococcus lactis. J Bacteriol 175, 7523-7532.

Wells, J. M., Wilson, P. W. \& Le Page, R. W. (1993). Improved cloning vectors and transformation procedure for Lactococcus lactis. J Appl Bacteriol 74, 629-636.

Edited by: V. Eijsink 